\title{
Type I,II and X Collagen Gene Expression Pattern in Bone Formation Using rh-BMP2
}

\author{
Naoki Katase' ${ }^{1)}$, Hitoshi Nagatsuka1), Yuzo Ishiwari ${ }^{1)}$, Hironobu Konouchi' ${ }^{1)}$ \\ Masao Inoue ${ }^{2)}$, and Noriyuki Nagai ${ }^{1)}$ \\ 1)Department of Oral pathology and Medicine, Graduate School of Medicine, Dentistry and Pharmaceutical Sciences, Okayama \\ University, Okayama, Japan \\ 2) Japan Institute for Advanced Dentistry, Osaka, Japan
}

\begin{abstract}
Bone morphogenic protein (BMP) induces ectopic bone. In that process, there are some tissue-specific collagens (type I, II and X) in osteocartilagenous matrix. And these types of collagen are also observed in the process of ossification in condylar cartilage. We searched for the localization of collagen gene expression in ectopic bone induction using rh-BMP and condylar cartilage ossification. BMP-type I collagen complex were implanted into the subcutaneous tissue of the dorsal region of ICR mice. Then we made specimens 3days, 1 week, and 2 weeks after implanting. For condylar cartilage, we pick out condylar region from ICR mice, and made specimens. The probes was made from mouse type I, II and X collagen cDNA segment (kikindly provided by Dr.Ninomiya of Okayama Universy). The process of ectopic bone induction using rh-BMP was the process through the intermediary of rapidly formed cartilagenoid tissue. And there are some difference of type I ,II and $\mathrm{X}$ collagen gene expressions between the process using rh-BMP and that of condylar cartilage. Those gene expressions imply that the ectopic hard tissue formative cells induced in early stage will take on the characters of osteoblasts and chondrocytes, and that the cells in growing cell layer in cartilagenoid tissue can differentiate into both osteoblasts and chondrocytes.
\end{abstract}

\section{Introduction}

Bone morphogenic protein (BMP) induces ectopic bone through means of the process similar to endochondral ossification. In that process, there are some tissue-specific collagens (type I, II and $\mathrm{X}$ ) in osteocartilagenous matrix. And type I, II and X collagens are also observed in the process of ossification in condylar cartilage. In this study, we search for the localization of collagen gene expression in ectopic bone induction using rh-BMP and condylar cartilage ossification by means of in situ hybridization, and compare the difference between the two.

\section{Material and method}

BMP-type I collagen complex, made from bovine metacarpal bone, were implanted into the subcutaneous tissue of the dorsal region of ICR male mice age in 3weeks. Then we made specimens 3days, 1week, and 2weeks after implanting. For condylar cartilage, we pick out condylar region from ICR male mice age of 1week, and made specimens. All specimens were stained with hematoxylin and eosin, and in situ hybridization was performed. For making probes, we used mice typeI and X collagen cDNA segment (kindly provided by Dr.Ninomiya of Okayama University).

\section{Results}

1. Ectopic bone induction using rh-BMP (Fig.1)

(1)3 days after implanting

Proliferation of spindle like cells is seen between the carrier and muscle. Carrier, Muscle,Spindle like cells The expression of collagen type I, II and X mRNA signals are seen in spindle like cells, chondrocyte-like cells and hypertrophic chondrocyte-like cells.

(2),2 week after implanting

Forming of cartilage like tissue is seen around the carrier.

The expression of collagen type I and II signals are still high in spindle like cells, chondrocyte-like cells, though that in hypertrophic chondrocyte-like cells is weakened. That of collagen type is strongly seen in hypertrophic chondrocyte-like cells, but that in chondrocyte-like cells disappeared.

\section{(3)2 weeks after implanting}

Hypertrophic chondrocyte-like cells $(\mathrm{H})$ are going to be encapsulated and opened. The expression of collagen type signals is seen in hypertrophic chondrocyte-like cells and osteoblast-like cells around the cartilage-like tissue and bone-like tissue. The expression of collagen type $\mathrm{X}$ signals is seen in encapsulated and opened hypertrophic chondrocyte-like cells.

\section{Condylar cartilage}

From surface to deep part, cartilage is divided into arthrogenous layer (A), Proliferating layer (P), Mature cell layer (M) and Hypertrophic cell layer $(\mathrm{H})$. The expression of collagen type I is seen in almost all layer.

The expression of collagen type II is seen in Proliferating layer (P), Mature cell layer (M) and Hypertrophic cell layer (H).The expression of collagen type II is seen in Mature cell layer (M) and Hypertrophic cell layer $(\mathrm{H})$.

The expression of collagen type I is seen in osteoblast-like cells surrounding the small piece of cartilage matrix.

The expression of collagen type $\mathrm{X}$ is seen in hypertrophic chondrocyte-like cells.

\section{Discussion and Conclusion}

The process of ectopic bone induction using rh-BMP was the process through the intermediary of rapidly formed cartilagenoid tissue. And there are some difference of type I, II and X collagen gene expressions between the process using rh-BMP and that of condylar cartilage. In addition, the collagen gene expression in both process implied that the cells in growing cell layer in 
International symposium of Maxillofacial \& Oral Regenerative Biology in Okayama 2005

cartilagenoid tissue can differentiate into both osteoblasts and chondrocytes.

There are some difference in the localization of collagen gene expression between ectopic bone induction using rh-BMP and condylar cartilage ossification.

The gene expressions of type I, II and Xcollagen imply that the ectopic hard tissue formative cells induced in early stage will take on the characters of osteoblasts and chondrocytes.

The collagen gene expression in both process implied that the cells in growing cell layer in cartilagenoid tissue can differentiate into both osteoblasts and chondrocytes.

\section{References}

1) Ishiwari $Y$ et al.:Localized expression of type I, II and $X$ collagen genes in the mandibular condyle of immature

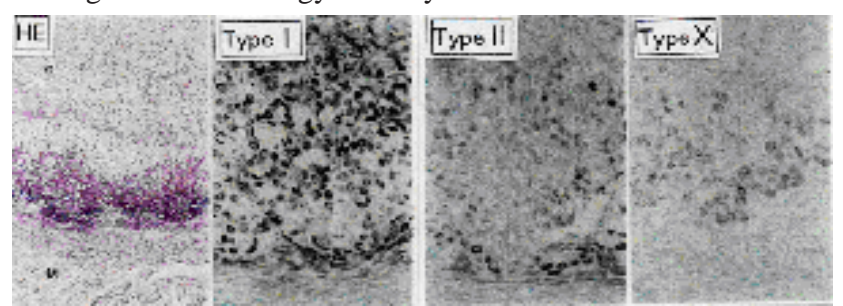

Fig.1 Ectopic bone induction using rh-BMP

mice.15,53-62,1996

2) Konouchi H: Gene expression of type I, II and X collagen during ectopic bone formation induced by bone morphogenetic protein 16,117-132,1997 